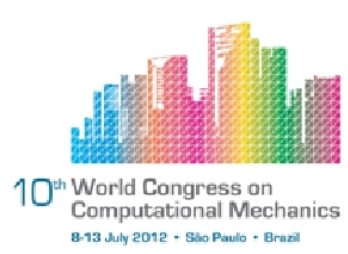

\title{
THERMO-MECHANICAL MODELING OF FRICTION STIR WELDING PROCESS VIA AN ITERATIVE MATRIX FREE GMRES DOMAIN DECOMPOSITION TECHNIQUE
}

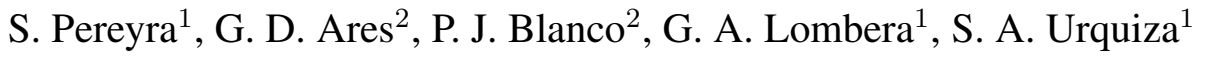 \\ ${ }^{1}$ Department of Mechanical Engineering, Engineering Faculty of Mar del Plata National Uni- \\ versity (spereyra@fi.mdp.edu.ar) \\ ${ }^{2}$ Laboratório Nacional de Computação Científica, Rio de Janeiro
}

\begin{abstract}
The friction stir welding (FSW) process consists essentially of a rotating tool, which travels along the joint of two restricted plates. The union results from the frictional heating induced by the tool, which leads to a viscoplastic material flow between the joined plates. A complete analysis of the FSW process requires the solution of a thermo-mechanical coupled problem. Due to the considerably large plastic deformation involved, an Eulerian viscoplastic flow formulation on a fixed domain is usually employed to mathematically describe the problem. Nevertheless, when the details of the tool must be taken into account the symmetry of the problem about the rotation axis of the pin is definitively lost. In this regard, an effective strategy to deal with this difficulty is to attach a rotating domain to the pin. Moreover, an arbitrary Lagrangian-Eulerian (ALE) formulation is used for irregular tool geometries, considering a convenient rotating domain to precisely track the details of the tool surface.

The purpose of this work is to implement a domain decomposition technique in order to exploit the advantages of both formulations. Hence, the whole domain is decomposed into two non-overlapping sub-domains in accordance with the particularities of each region. Consequently, a rotating region close to the tool is adopted while a fixed domain surrounding it is considered to appropriately take into account the inlet and outlet boundary conditions. Thereby, a coupling problem on the common boundary to both sub-domains must be solved. In this work a coupling strategy based on an iterative GMRES technique in its matrix free form is adopted. The application of this methodology allows to determine the material flow and temperature field considering the specific details of the tool and welding plates geometries in an efficient and easy to implement manner.
\end{abstract}

Keywords: Friction Stir Welding, coupling domains, Matrix free coupling.

\section{INTRODUCCION}

Friction Stir Welding (FSW) es un proceso de soldadura de estado sólido, es decir, el material en la unión no supera su temperatura de fusión durante el proceso. Se emplea una 
herramienta cilíndrica provista de un perno en uno de sus extremos, definiéndose en el lugar donde se encuentra el perno con el cuerpo de la herramienta un hombro o apoyo.

El proceso comienza con la herramienta girando y la introducción del perno de la herramienta entre la línea de unión de las piezas a soldar hasta que el hombro hace contacto con las superficies de las mismas. A continuación se hace avanzar a la herramienta a lo largo de la línea de unión sin interrumpir su giro. La gran fricción entre las superficies de la herramienta en contacto con el material de las piezas en conjunto con la gran deformación plástica del material de las piezas alrededor de la herramienta produce un aumento de la temperatura. En consecuencia el material se ablanda y el mismo movimiento de la herramienta produce un flujo de material que resulta en la unión de las piezas.

En la Figura 1 se muestra un esquema del proceso de soldadura.

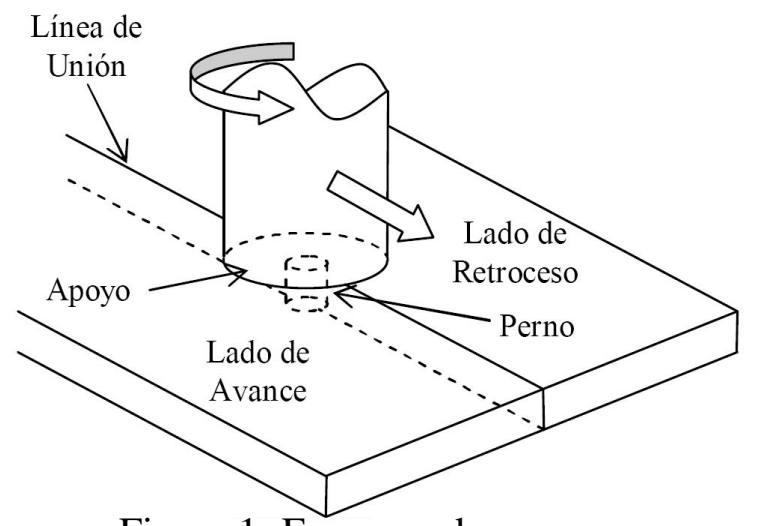

Figure 1. Esquema de proceso.

La herramienta puede tener diferentes formas geométricas que afectan al flujo de material. Las herramientas convencionales mencionadas en la literatura están provistas de pernos cilíndricos roscados y apoyo u hombros cóncavos. Además, se han desarrollado herramientas con geometrías más complejas, tales como las Whorl ${ }^{\mathrm{TM}}$, Triflute ${ }^{\mathrm{TM}}$, Triflat $^{\mathrm{TM}} \mathrm{y}_{\text {Trivex }^{\mathrm{TM}}}[10]$. Entender en forma completa el transporte de material alrededor de la herramienta es fundamental para poder encontrar el conjunto de variables de proceso (las velocidades de rotación y traslación de la herramienta, el ángulo de inclinación del eje de la herramienta, y la profundidad de penetración) que permitan obtener uniones con las características requeridas. En este sentido, los modelos computacionales en conjunto con técnicas experimentales, tales como las técnicas de insertos marcadores, etc. [1, 8, 7], son necesarios para poder comprender como afectan las anteriores variables de proceso al patrón del flujo de material durante la soldadura.

Existe una gran cantidad de trabajos de modelado numérico dirigidos a determinar el flujo de material en uniones soldadas por FSW. Los modelos de flujo convencionales, con descripción Eulerina, han servido para casos que incluyen herramientas con geometría axisimétrica [11] e incluso se ha tenido en cuenta la presencia de la rosca a través de condiciones de borde particulares sobre la herramienta [5]. Sin embargo, estos modelos no permiten considerar geometrías complicadas. Para poder enfrentar estos casos se consideran los modelos con descripción Lagrangeana Eulerina Arbitraria (ALE) $[2,6]$.

Este tipo de modelos con descripción ALE implican la resolución sobre una geometría que gira solidaria a la herramienta, por lo que las piezas a soldar deben tener una forma circular. En la práctica, la soldadura se efectúa sobre piezas que pueden tener distintas formas 
y las condiciones de borde impuestas sobre los contornos circulares no son adecuadas para representarlas. En este sentido, es posible considerar una geometría fija externa acoplada de algún modo con la geometría que gira. Teniendo en cuenta que el problema se pretende resolver por medio del Método de los Elementos Finitos (FEM), se requeriría actualizar la conectividad de los elementos cuyos nodos coincidan con los nodos de la otra malla en la frontera de acople. Una alternativa a este procedimiento, es considerar el acople a través de alguna de las técnicas de descomposición de dominios, es decir, resolver cada dominio en forma separada considerando la interacción de los dominios a través de condiciones de borde. La técnica Neumann-Neumann implica imponer como condición de borde sobre la frontera de acople correspondiente a cada malla flujos de calor y fuerzas nodales obteniéndose valores de temperatura y velocidad. Las técnicas de tipo Dirichlet-Dirichlet o Neumann-Dirichlet conllevan la complicación de considerar todos los elementos que están conectados a cada nodo de la frontera de acople para poder efectuar el cálculo de los flujos de calor y fuerzas nodales.

Se aplicará una técnica descomposición de dominios para acoplar una malla fija, con descripción Euleriana, y otra móvil, con descripcin ALE, correspondientes a un modelo termomecánico bidimensional del proceso de soldadura por friccin FSW.

\section{MODELOS NUMERICOS}

\subsection{Modelo mecánico}

Las ecuaciones de equilibrio en un volumen de material $\Omega$ con una frontera $\partial \Omega$, despreciando las fuerzas inerciales y de volumen, se pueden escribir de la siguiente manera [12]:

$$
\frac{\partial \sigma_{k l}}{\partial x_{l}}=0 \text { en } \Omega
$$

donde $\sigma_{k l}$ es el tensor de tensiones de Cauchy que, de acuerdo con la formulación de flujo adoptada, adquiere la forma:

$$
\sigma_{k l}=-p \cdot \delta_{k l}+\tau_{k l}
$$

donde $\delta_{k l}$ es la matriz identidad, $p$ es la presión hidrostática y $\tau_{k l}$ es el tensor desviador de tensiones. Este último puede relacionarse con el tensor de velocidades de deformación $d_{k l}$ por medio de la viscosidad $\mu$, segn la siguiente relación:

$$
\begin{gathered}
\tau_{k l}=2 \cdot \mu \cdot d_{k l}, \\
d_{k l}=\frac{1}{2} \cdot\left(\frac{\partial u_{k}}{\partial x_{l}}+\frac{\partial u_{l}}{\partial x_{k}}\right), \\
\mu=\frac{\sigma_{\mathrm{f}}}{3 \cdot \dot{\varepsilon}_{\mathrm{e}}},
\end{gathered}
$$

donde $\sigma_{\mathrm{f}}$ es la tensión efectiva o el segundo invariante de la tensión y $\dot{\varepsilon}_{\mathrm{e}}$ es la velocidad de deformación efectiva o el segundo invariante de la velocidad de deformación, que puede obtenerse por 


$$
\dot{\varepsilon}_{\mathrm{e}}=\sqrt[2]{\frac{2}{3} \cdot d_{k m} \cdot d_{k m}}
$$

Se asume que el material tiene un comportamiento viscoplástico y rígido por lo que la tensión de flujo depende de la velocidad de deformación y de la temperatura y puede representarse por la siguiente relación constitutiva propuesta por Sheppard et al. [9]:

$$
\begin{gathered}
\sigma_{\mathrm{f}}=\frac{1}{\alpha} \cdot \sinh ^{-1}\left[\left(\frac{Z}{A}\right)^{\frac{1}{n_{c}}}\right], \\
Z=\dot{\varepsilon}_{\mathrm{e}} \cdot \exp \left(\frac{Q}{R \cdot \theta}\right),
\end{gathered}
$$

donde $\alpha, Q, A$ y $n_{c}$ son constantes del material (ver Tabla 1), $R$ es la constante de los gases y $\theta$ es la temperatura absoluta. Las constantes del material se pueden determinar usando ensayos de compresin estándar.

Para completar el modelo mecánico, se especifican las condiciones de contorno apropiadas: las tracciones $t_{i}{ }^{0}$ en una porción de la frontera $\partial \Omega_{t}$ (condiciones de Neumann) y las componentes de la velocidad $u_{k}{ }^{0}$ en el resto de la frontera $\partial \Omega_{u}$ (condiciones de Dirichlet). Esto se puede expresar como:

$$
\begin{gathered}
\sigma_{k l} \cdot n_{l}=t_{k}^{0} \text { en } \partial \Omega_{t}, \\
u_{k}=u_{k}{ }^{0} \text { en } \partial \Omega_{u},
\end{gathered}
$$

donde $\partial \Omega=\partial \Omega_{t} \cup \partial \Omega_{u}$.

Table 1. Parámetros de la ecuación para la tensión de flujo

\begin{tabular}{ccccc}
\hline Material & $A$ & $\alpha$ & $n_{c}$ & $Q$ \\
\hline Alloys 1S & $0.224 \cdot 10^{13}$ & $0.05 \mathrm{~mm}^{2} \mathrm{~N}^{-1}$ & 4.54 & $177876.4 \mathrm{~J} \mathrm{~mol}^{-1}$ \\
\hline
\end{tabular}

\subsection{Modelado térmico}

La ecuación de balance de calor en un volumen de material $\Omega$ con una frontera $\partial \Omega$ es:

$$
\rho \cdot C p \cdot u_{k}^{r e f} \cdot \frac{\partial \theta}{\partial x_{k}}=-\frac{\partial}{\partial x_{l}}\left(-K \cdot \frac{\partial \theta}{\partial x_{l}}\right)+\rho \cdot \gamma \text { en } \Omega
$$

donde $C p$ es la capacidad calorífica, $K$ es la conductividad térmica, $\theta$ es la temperatura, $u_{k}^{r e f}$ es la velocidad del material respecto al sistema de referencia y $\gamma$ es la tasa de generación de calor interna por disipación de la potencia viscosa. Se asume que alrededor del 0.90 de la potencia plástica se convierte en calor [3]. El término de tasa de generación de calor por trabajado mecánico se puede expresar como el producto contraído de la tensión con la velocidad de deformación, como sigue:

$$
\gamma=\eta \cdot \tau_{k l} \cdot d_{k l}
$$


con $\eta$ fracción de potencia que no es absorbida en defectos microestructurales.

Además se adoptaron las propiedades del aluminio puro, tanto para los parámetros mecánicos como para la conductividad y el calor específico dependientes de la temperatura. Sobre una porción del contorno $\partial \Omega_{q}$ se prescriben los flujos de calor $q_{k}{ }^{0}$, mientras que en el resto del contorno $\partial \Omega_{\theta}$ se especifica la temperatura $\theta$. Esto se puede expresar como:

$$
\begin{gathered}
-K \cdot \frac{\partial \theta}{\partial n}=q^{0} \text { en } \partial \Omega_{q}, \\
\theta=\theta^{0} \text { en } \partial \Omega_{\theta},
\end{gathered}
$$

donde $\partial \Omega=\partial \Omega_{q} \cup \partial \Omega_{\theta}$ y $n$ es el versor normal al contorno $\partial \Omega_{q}$.

\section{DESCOMPOSICION DE DOMINIOS}

La presente técnica de descomposición está concebida particularmente para llevar a cabo el acoplamiento de los modelos correspondientes a las mallas móvil y fija, resolviendo cada submodelo por separado e implementando la interacción entre los subdominios sólo por medio de condiciones de borde.

Siguiendo las ideas básicas de la técnica de descomposición de dominios, se consideran las ecuaciones de gobierno en una región $\Omega$, en dos o tres dimensiones, con condiciones de borde (Dirichlet o Neumann) en la frontera $\partial \Omega$ de $\Omega$. Se supone también que $\Omega$ se particiona en dos subdominios $\Omega_{i}$ que no se superponen (ver Figura 2):

$$
\Omega=\Omega_{1} \cup \Omega_{2}, \Omega_{1} \cap \Omega_{2}=\varnothing, \Gamma=\partial \Omega_{1} \cap \partial \Omega_{2} .
$$

Se define de esta manera una frontera de acople $\Gamma$ con condiciones de borde asociadas, llamadas condiciones de transmisión (transmission conditions).

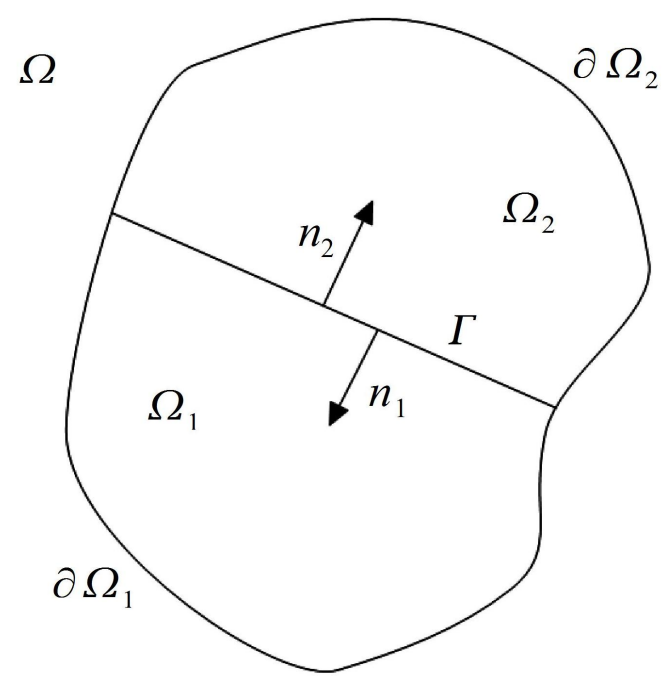

Figure 2. Acople de dominios.

La presente técnica de acople puede verse como variante del algoritmo clásico NeumannNeumann en el cual cada uno de los dominios recibe los mismo valores de flujos y fuerzas. 
Llamando $u_{j}{ }^{1}, \theta^{1}, u_{j}{ }^{2}$ y $\theta^{2}$ a las temperaturas y velocidades de los nodos, obtenidas por medio del cálculo computacional, correspondientes a $\partial \Omega_{1}$ y a $\partial \Omega_{2}$, respectivamente, y $u_{j}{ }^{0}$ y $\theta^{0}$ a las velocidades y temperaturas, y $q^{0}$ y $t_{j}{ }^{0} \operatorname{los}$ flujos de calor y fuerzas, incógnitas de problema, sobre la frontera de acople $\Gamma$, debe cumplirse lo siguiente:

$$
\begin{gathered}
u_{j}{ }^{1}\left(t_{j}{ }^{0}\right)-u_{j}{ }^{0}=0, \\
\theta^{1}\left(q^{0}\right)-\theta^{0}=0, \\
u_{j}^{2}\left(t_{j}{ }^{0}\right)-u_{j}^{0}=0, \\
\theta^{2}\left(q^{0}\right)-\theta^{0}=0 .
\end{gathered}
$$

Teniendo en cuenta que se desconocen tanto $u_{j}{ }^{0}$ y $\theta^{0}$ como $t_{j}{ }^{0}$ y $q^{0}$, a partir de las ecuaciones 16, 17, 18 y 19 se establecen cuatro residuos que definen el problema a resolver de la siguiente forma:

$$
\begin{gathered}
r_{k}{ }^{1}\left(u_{j}{ }^{0}, t_{j}{ }^{0}\right)=u_{j}{ }^{0}-u_{j}{ }^{1}\left(t_{j}{ }^{0}\right), \\
r_{k}{ }^{1}\left(\theta^{0}, q^{0}\right)=\theta^{0}-\theta^{1}\left(q^{0}\right), \\
r_{k}{ }^{2}\left(u_{j}{ }^{0}, t_{j}{ }^{0}\right)=u_{j}{ }^{0}-u_{j}{ }^{2}\left(t_{j}{ }^{0}\right), \\
r_{k}{ }^{2}\left(\theta^{0}, q^{0}\right)=\theta^{0}-\theta^{2}\left(q^{0}\right) .
\end{gathered}
$$

Dado que los residuos en conjunto resultan en un sistema de ecuaciones no lineales, se considera el resolvedor GMRES no marticial (matrix-free), que es un método iterativo basado en subespacios de Krylov, para su resolución.

\section{DESCRIPCION DEL MODELO}

Para las piezas a soldar se considera un dominio bidimensional que se encuentra limitado por los bordes externos de las piezas a soldar y por el contorno del perno roscado de la herramienta (ver Figura 3). Este último corresponde al corte del perno, transversal al eje de la herramienta, que varía según cambia la posición angular de la herramienta a medida que gira. En línea de trazos se encuentran marcadas las circunferencias correspondientes al diámetro interior y exterior que limitan las distintas posiciones en cuestión.

El dominio se divide en dos: un dominio fijo $\Omega_{2}$, con descripción Euleriana, y otro móvil $\Omega_{1}$, con descripción ALE. Ambos dominios se encuentran vinculados a través de una frontera de acople $\Gamma$. A su vez, en el dominio móvil $\Omega_{1}$ se diferencian dos zonas: cercana $\Omega_{1}{ }^{C}$ y lejana $\Omega_{1}{ }^{L}$. Esta diferenciación se debe al distinto tratamiento del transporte asociado al movimiento de la malla correspondiente al modelo térmico sobre cada una de las zonas [6]. 

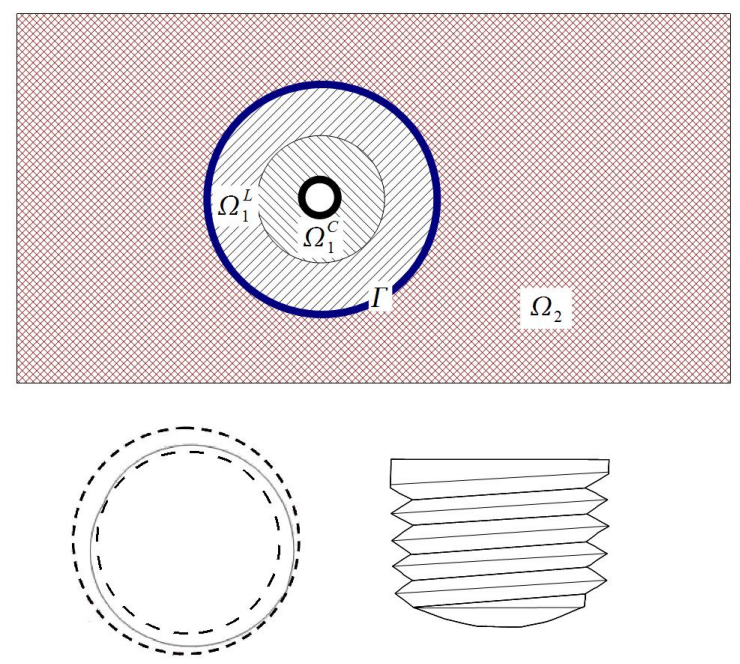

Figure 3. Geometría y división del dominio.

Para la primera se efectúa un transporte exacto y para la última una separación en dos etapas (operator splitting).

La malla está conformada por 4,148 elementos y 6,748 nodos, con 56 nodos en la frontera de acople. Respecto al resolvedor iterativo GMRES, se considero un subespacio de Krylov de dimensión 12.

\section{RESULTADOS}

En la Figura 4 se muestra la distribución de temperaturas del dominio bidimensional.
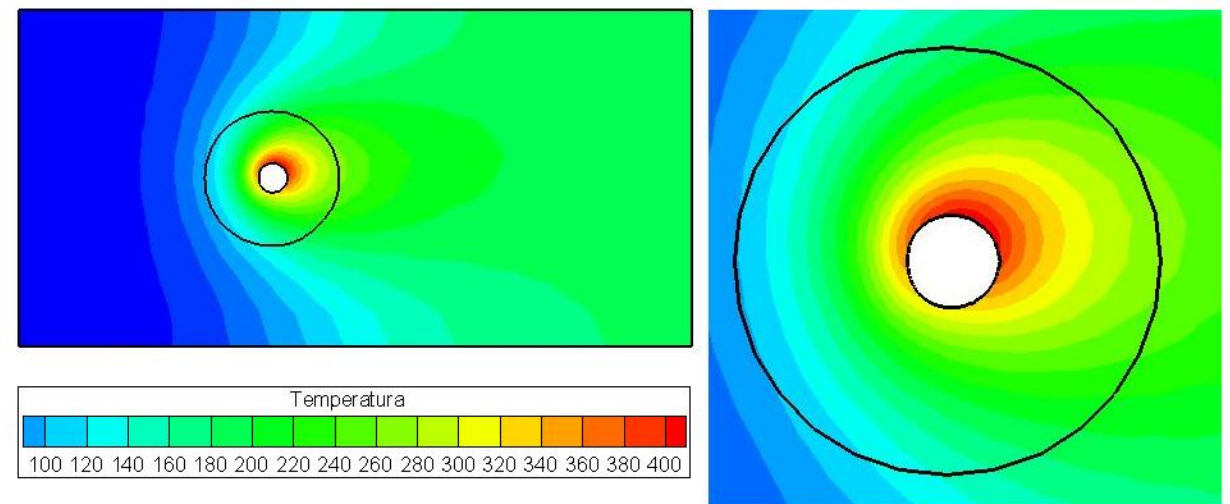

Figure 4. Campo de temperaturas.

Por un lado, se observa que, sobre la frontera de acople, la temperatura de ambos dominios coincide. Por otro lado, se ve que si se hubiera tomado una geometría circular para las piezas a soldar el resultado cambiaría, ya que las isotermas sobre el contorno externo estarían dirigidas hacia el eje de la herramienta.

En la Figura 5 se muestran las líneas de corriente del material.

De la misma forma, las líneas de corriente de material atraviesan sin discontinuidades la frontera de acople entre los dominios. 


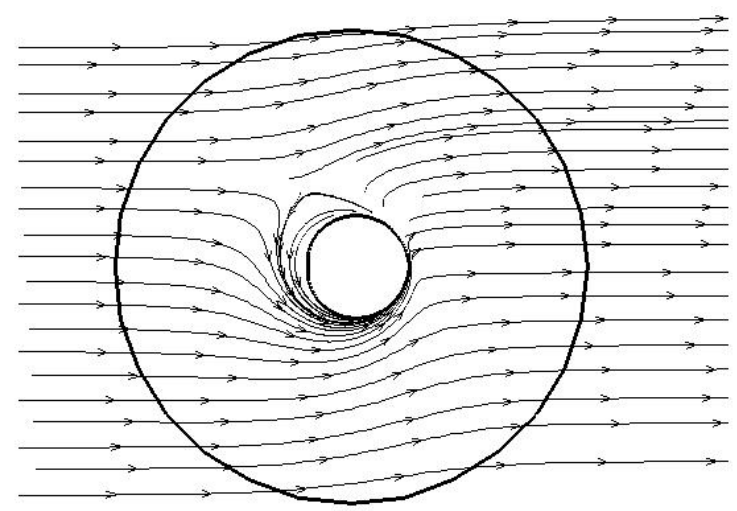

Figure 5. Líneas de corriente.

\section{CONCLUSIONES}

Se resolvió un modelo bidimensional simplificado inspirado en el proceso de soldadura por fricción FSW considerando una frontera de acople entre una malla fija, con descripción Euleriana, y una malla móvil, con descripción Euleriana-Lagrangeana Arbitraria. La técnica de acople empleada resultó adecuada para aplicarse a la resolución de este tipo de problemas.

Los modelos simplificados resultan adecuados para extraer conclusiones comparando las diferentes respuestas a ciertas variaciones, e.g. modificando la cantidad de nodos en la frontera de acople o la dimensión del subespacio de Krylov, antes de extender el análisis a modelos tridimensionales.

Por otra parte, es necesario continuar con la búsqueda de nuevas posibilidades para esta técnica, teniendo en cuenta que permite la resolución del problema en forma paralela y el tratamiento de cada dominio en forma particular. 


\section{REFERENCIAS}

[1] Colligan K, "Material flow behavior during friction stir welding of aluminum". Welding Reserch Supplement. 229-237, 1999.

[2] Colegrove P. A., Shercliff H.R., "3-Dimensional CFD modelling of flow round a threaded friction stir welding tool profile". Journal of Materials Processing Technology. 169, 320327, 2005.

[3] Johnson W., Kudo H., "The Mechanics of Metal Extrusion". Manchester University Press. Manchester, United Kingdom, 1962.

[4] Saad Y., Schultz M.H., "GMRES: A Generalized Minimal Rresidual Algorithm for solving nonsymmetric linear sistems". Journal on Scientific and Statistical Computing. 7, 3:856-869, 1986.

[5] Santiago D., Pereyra S., Lombera G., Urquiza S., "Analisis de defectos en soldadura por fricción-agitación mediante un modelado 3D”. Mecánica Computacional 25, 2217-2226, 2006.

[6] Santiago D., Pereyra S., Lombera G., Urquiza S., "Modelado termomecánico del proceso friction stir welding utilizando la geometría de herramienta real". Mecánica Computacional. 28, 1673-1688, 2009.

[7] Schmidt H.N.B., Dickerson T.L., Hattel J.H., "Material flow in butt friction stir welds in AA2024-T3”. Acta Materialia. 54, 1199-1209, 2006.

[8] Seidel T. U., Reynolds A. P., "Visualization of the material flow in AA2195 Frictio Stir Welds using a marker insert technique". Metallurgical and Materials Transaction A. 32, 2879-2884, 2001.

[9] Sheppard T., Wright D. S., "Determination of flow stress: Part 1 constitutive equation for aluminum alloys at elevated temperatures". Metals Technology. 215, 1979.

[10] Threadgill P. L., Leonard A. J., Shercliff H. R., Withers P. J., "Friction stir welding of aluminium alloys". International Materials Reviews. 54, 2:49-93, 2009.

[11] Ulysse P., "Three-dimensional modeling of the friction stir-welding process". International Journal of Machine Tools and Manufacture. 42, 1549-1557, 2002.

[12] Zienkiewicz O.C., Taylor R.L., "The finite element method. Volume II". McGraw Hill. 1991. 